\title{
Neurofeedback Versus Pharmacological Intervention in the Treatment of Childhood Attention Deficit/Hyperactivity Disorder (ADHD): First Spanish Clinical Neuropsychological Study
}

\author{
Denise Medici $^{1,5, \text { * }}$, Pau Giner Bayarri², Rosa Chilet Chilet ${ }^{2}$, Juan Moliner Ibánez ${ }^{2}$, \\ Maria Morales Suarez-Varela ${ }^{1,3,4}$, , Dayana Calvo ${ }^{6}$ \\ ${ }^{1}$ Department of Preventive Medicine, University of Valencia, Valencia, Spain \\ ${ }^{2}$ Department of Neurophysiology, Hospital Universitario Dr. Peset, Valencia, Spain \\ ${ }^{3}$ Department of Biomedical Research Consortium for Epidemiology and Public Health (CIBERESP), Valencia, Spain \\ ${ }^{4}$ Center for Public Health Research (CSISP), Valencia, Spain \\ ${ }^{5}$ Department of Neuropsychology, Hospital Vithas Nisa 9 de Octubre, Valencia, Spain \\ ${ }^{6}$ Department of Neuropsychology, James A. Haley Veteran's Hospital, Tampa, USA \\ Email address: \\ denise_medici@yahoo.es (D. Medici), maria.m.morales@uv.es (M. M. Suarez-Varela) \\ ${ }^{*}$ Corresponding author
}

\section{To cite this article:}

Denise Medici, Pau Giner Bayarri, Rosa Chilet Chilet, Juan Moliner Ibánez, Maria Morales Suarez-Varela, Dayana Calvo. Neurofeedback Versus Pharmacological Intervention in the Treatment of Childhood Attention Deficit/Hyperactivity Disorder (ADHD): First Spanish Clinical Neuropsychological Study. American Journal of Applied Psychology. Vol. 7, No. 5, 2018, pp. 57-66. doi: 10.11648/j.ajap.20180705.11

Received: October 3, 2018; Accepted: November 6, 2018; Published: December 18, 2018

\begin{abstract}
In this study, twenty children diagnosed with ADHD according to the Diagnostic and Statistical Manual of Mental Disorders, Fourth Edition criteria (DSM-IV) were randomly assigned to receive NFB training or MPH treatment. The participants were evaluated at the beginning of the study and four months later on measures of behavioral (CPRS/CPRT), executive functions (BRIEF, CPT), attention (TOVA), and electrical activity (qEEG, ERPs). Post-intervention results showed improvements in attention, hyperactivity, executive functioning and in continuous performance measurement (CPT).Improvements noted in the NFB group were greater than that of the MPH group. Results of this study suggest NFB training resulted in greater improvements in executive functioning, behavior, attention, and qEEG compared to MPH treatment. Results indicate that neurofeedback may treatcognitive and behavior functions before these functions worsen or decrease. This study suggests future research to compare the efficacy of each of these treatments in larger populations with a greater heterogeneity in gender is warranted.
\end{abstract}

Keywords: ADHD, Quantitative Electroencephalogram, Event-related Potentials, Continuous Performance Test, Neurofeedback, Methylphenidate

\section{Introduction}

Attention Deficit/Hyperactivity Disorder (ADHD) is the most common behavioral problem encountered by pediatricians in primary care settings and has a worldwidepooled prevalence was 5.29\% [1] (Polanczyk, de Lima, Horta, Biederman, \& Rohde, 2007). Several studies have found that ADHD subjects had more lifetime psychopathology. Male gender increases risk for disruptive behavior disorders. Female gender and oppositional defiant disorder contributed to risk for depression and anxiety. They had more parent-reported problems in terms of emotionalbehavioral role function, behavior, mental health, and selfesteem [2] (McGough, Smalley, \& McCracken, 2005). The problems of children with ADHD show a significant impact on the parents' emotional health and parents' time to meet 
their own needs, and they interfered with family activities and family cohesion [3] (Klassen, Miller, \& Fine, 2004).

In the childhood ADHD population, disturbances can include: behavioral disorders (30-50\%), oppositional defiance (15-75\%), anxiety (25-50\%), mood alterations (15$75 \%$ ), and co-morbid learning disorders (8-39\%) [4] (Monastra, Lynn, Linden, Lubar, Gruzelier, \& LaVaque, 2005). Co-morbid learning disorders can include mathematics (12-30\%), written expression (30-50\%) and spelling (12-27\%) [5] (Monastra, 2005).

ADHD in children is typically treated with stimulants medication. The Methylphenidate (MPH) is currently the first-line pharmacological treatment for children [6] (Myer, Boland \& Faraone, 2017) has been show to improved spatial working memory (WM), attention-set shifting and visualsearch task performance [7] (Metha, Goodyer, \& Sahakian. 2004). However results from a recent meta-analysis highlight the importance of carefully weighing the potential benefits and adverse effects of stimulant medications when prescribing to children. Another important aspect is the sleep impairment because is related to many cognitive (e.g., inattention) and emotional/behavioral (i.e., defiant, anger) and sleep adverse effects could undermine the benefits of stimulant medications in some cases [8] (Kidwell, Van Dyk, Lundahl, \& Nelson, 2015). Further, pharmacological treatment works only while it is present in the patient's cerebral system, so ADHD symptoms return after pharmacological management has ended [9] (Barkley, Fischer, Fletcher, \& Smallish, 2004). Thus, alternative effective treatments for ADHD are desired.

Neurofeedback (NFB) training has been proposed as anon invasive method for treatment of neurobehavioral symptoms in ADHD, and to date, there are no know negative side effects [5] (Monastra, 2005). NFB involves recording electrical activity in the brain and transforming it into a digital visual and/or auditory signal that is utilized as feedback to teach the patient to self-regulate the amplitude of specific frequency waves [10] (Hammond, 2011). A significant clinical improvement was reported in nearly $75 \%$ of the children treated with NFB [4] (Monastra, Lynn, Linden, Lubar, Gruzelier, \& LaVaque, 2005). NFB training seems to have long-lasting effects because learning is internalized [11] (Lubar, 1997). Evidenced of NFB's effectiveness in improving attention and impulsive behavior [12-15] (Arns, de Ridder, Strehl, Breteler, \& Coenen, 2009; Magee, Clarke, Barry, McCarthy, \& Selikowitz, 2005; Rossiter, 2004; Bluschke, Broschwitz, Kohl, Roessner, \& Beste, 2016), and ADHD symptoms [16-21] (Beauregard, \& Lévesque, 2006; Fuchs, Birbaumer, Lutzenberger, Gruzelier, \& Kaiser, 2003; Kropotov, Grin-Yatsenko, Ponomarev, Chutko, Yakovenko, \& Nikishena, 2005; Lubar, Swartwood, Swartwood, \& O'Donnell, 1995; Nash, 2000; Thompson, \& Thompson, 1998) has been documented. In these studies, the effects of NFB were very specific for situations requiring inhibitory control over responses [15] (Bluschke, Broschwitz, Kohl, Roessner, \& Beste, 2016).

In a recent review, the American Psychiatric
Electrophysiological Association summarized the evidence found for clinical utility of quantitative electroencephalogram (qEEG) in dementia [22, 23] (Fahimi, Tabatabaei, Fahimi, \& Rajebi, 2017; Garn, Coronel, Waser, Caravias, \& Ransmayr, 2017), mood disorders [24, 25] (Haghighi, et al., 2017; Khaleghi, Sheikhani, Mohammadi, \& Moti Nasrabadi, 2015), mild head injuries [26] (Thatcher, 2000), learning disabilities [27] (Arns, Peters, Breteler, \& Verhoeven, 2007), attention disorders [28-35] (Clarke, Barry, McCarthy, \& Selikowitz, 2001, 2001b, 2001c, 2001d; Clarke, Barry, McCarthy, Selikowitz, \& Brown, 2002; Lazzaro, Gordon, \& Whitmont, 2000; Hobbs, Clarke, Barry, McCarthy, \& Selikowitz, 2007; Linden, Habib, \& Radojevic, 1996) and psychosis [36] (Fuggetta, Bennett, Duke, \& Young, 2014). QEEG allows the determination of EEG differences between children with and without ADHD. Electrophysiological studies have provided consistent evidence for several abnormal oscillations during the resting states in patients with ADHD and the frontal inhibitory system has been implicated in problems with inhibitory regulation. [37] (Barry, Johnstone, \& Clarke, 2003). Children with ADHD generally demonstrated qEEG findings characterized by excess of slow theta and/or slow alpha peak performance in the fronto-parietal regions, which might lead to the finding of increased "theta" power [33, 38, 39] (Lazzaro, Gordon, \& Whitmont, 2000; Vernon, Egner, Cooper, Compton, Neilands, Sheri, \& Gruzelier, 2003; Mann, Lubar, Zimmerman, Millar, \& Muenchen, 1992). Other studies have identified greater absolute delta and theta activity and an increased theta/beta ratio as compared to controls [15, 34, 40, 41] (Bluschke, Broschwitz, Kohl, Roessner, \& Beste, 2016; Hobbs, Clarke, Barry, McCarthy, \& Selikowitz, 2007; Zhang, et al., 2017; Loo, \& Scott, 2012). Negative correlations between coherence anomalies in qEEG and ADHD symptoms suggest that several anomalies reflect compensatory brain function, and studies have reported reduced frontal coherence in delta, alpha and gamma waves in patients with ADHD [42] (Barry, Clarke, Hajos, Dupuy, McCarthy, \& Selikowitz, 2011). Moreover, coherence differences in ADHD had been may reflect anomalous frontal right-hemisphere linkages that help compensate functional brain anomalies in the left frontal regions. The slighter leftlateralized coherences correlated negatively with the DSMIV Inattentive and DSM-IV Total scores, and a slighter frontal inter-hemispheric coherence in alpha correlated negatively with the DSM Hyperactive/Impulsive Score [42] (Barry, Clarke, Hajos, Dupuy, McCarthy, \& Selikowitz, 2011). One recent work related resting-state EEG anomalies in individuals with ADHD, to their symptom profile and could indicate links between increased inattention scores and reduced resting EEG gamma power. With resting-state EEG coherence, reduced left lateralized coherences across several bands was correlated negatively with inattention scores, while reduced frontal interhemispheric coherence was correlated negatively with hyperactivity/impulsivity scores [43] (Barry, \& Clarke, 2013). Finally, the event-related potential (ERP) is a measured brain response that is a direct 
result of a specific cognitive event. ERPs have revealed important findings on the background pathologies of children with ADHD [37, 44] (Barry, Johnstone, \& Clarke, 2003; Kröger, et al., 2014).

The current study sought to evaluate effects of physiological self-regulation (i.e., NFB) in behavior, cognition, and qEEG and ERP finding in children with ADHD as compared to treatment by stimulant medication (MPH).

\section{Methods}

\subsection{Participants}

All patients in the Pediatric Department of the Hospital Universitario Dr. Peset who had been diagnosed with ADHD invited to participate in the current study. ADHD was diagnosed by a pediatrician using a detailed physical examination and clinical interview. The study was supported by the Consellería de Sanitat i Salut Publica of Valencia (DOCV 6507, 26/04/2011) and was conducted in keeping with the Declaration of Helsinki (World Medical Association, 2013, October). Ethics Committee: 10/061 and 11/083.

In the first phase, 20 children (18 males and 2 females) with diagnosed ADHD (10 children per group) participated in the study. Participants had not received any therapeutic and/or pharmacological treatment. Patients were randomized to either NFB or MPH group. In the NFB group (8 males and 2 females), one patient discontinued. The MPH group also started with 10 children, however, two children discontinued due to medication side effects. The participants were required to meet the following inclusion criteria: age 8 to 14 years old; a primary formal diagnosis of ADHD based on a semistructured interviews with their parents using DSM-IV in the Pediatric Department; no diagnosis of comorbid neurological disorders (epilepsy, traumatic brain injury, Tourette's syndrome); no serious medical conditions; no use of psychostimulants or atomoxetine medication treatment before the study; an intellectual quotient $\geq 80$; normal EEG results; and a well-structured family, referring to families with the following characteristics: parents who do not obstruct child motivation, perfect attendance at the NFB sessions, and families with a sense of responsibility, because this condition could have an equally strong impact.

Children were excluded from participation if they failed to meet any of these requirements, or if they had serious medical conditions, EEG with abnormal results or brain injury, seizures or epileptic disorders.

\subsection{Materials and Procedure}

We had access to all records from the diagnoses made by the Pediatric Department and from academic reports. Neither group showed statistically significant differences as between social, educational, family conditions. referring to children from the same neighborhood and similar public schools, and the parents with similar income and studies between Junior and High School.
Prior to the study's initiation, parents of the subjects were informed about their rights and provided with a written informed consent. In the informed consent form, permission was requested to use the participant's neurophysiologic and neuropsychological data for the specified scientific research. The importance of parental support during the four months in both groups was emphasized as a vital consideration for participating. We made a previous stipulation about the importance of parents' support and the probability of perfect attendance for the next four months. This was a random assignment process.

No participant was allowed to leave the treatment during the study.

After considering the diagnostic usefulness of EEG, we obtained more measures using rigorous diagnostic procedures (i.e., structured diagnostic interviews, neuropsychological assessments) and for careful identification of co-morbid diagnoses (including specific learning disorders) and the impact of these disorders on EEG characteristics (see Table 1). The measures in both groups when we started and finished the study were the following: qEEG and ERPs assessment data collection, Parent and Teacher Conners' Rating Scale (CPRS \& CTRS in Short Version, Behavior Rating Inventory of Executive Functions Parent and Teacher Form (BRIEF), and Test of Variables of Attention (TOVA).

Pre-treatment screening and baseline neuropsychological measurements

Both scales were rated by parents and teachers.

The Behavior Rating Inventory of Executive Function (BRIEF) parent and teachers rating form contains 86 items in eight non-overlapping clinical scales. These theoretically and statistically derived scales form two indexes: a) Behavioral Regulation (three scales) and b) Metacognition (five scales), as well as a Global Executive Composite score which takes into account all of the clinical scales and represents the child's overall executive function [45].

The Conners 3rd Edition-Parent and Teachers Short form (CPRs and CTRs) is an assessment tool used to obtain the parent's and teacher's observations about the youth's behavior. This short version provides evaluation of the key areas of inattention, hyperactivity/impulsivity, learning problems, executive functioning, aggression, and peer relations. Each of the items on the scale is rated using four categories: "not at all," just a little, "much," and "very much" [46] (Conners, 2007).

Finally, children meeting the clinical criteria for inclusion in the study were evaluated on pre and post both treatments with the TOVA. It is a computer administered/scored test of attention abilities and is considered one of the most widely used measures of attention and impulsivity. These Continuous Performance Tests (CPTs) provide an assessment of an individual's performance on a task that requires tracking of visual stimuli with differential response/nonresponse to target and nontarget stimuli. Scores obtained included errors of inattention (i.e., failure to respond to a target stimulus or absence of a response when one is required and it is called error of omission) and impulsivity 
(i.e., response to a nontarget stimuli or when no response was required and it is called error of commission), response time rate and the consistency of response rate (variability). The duration of testing is 21.8 minutes long in each sensory modality (visual and auditory) [47] (Greenberg, Kindschi, \& Corman, 2000).

The behavioral scale, the executive functions scale and the two CPT's modalities are reliable markers of executive brain functioning.

Participants were instructed to quietly while the qEEG was recorded on a Neuronic ${ }^{\circledR}$ Medicid using with 36 electrodes (according to the 10-20 electrode international system) for 5 minutes with eyes closed and 30 minutes with eyes open in resting-state conditions. Data were referenced to a common reference placed between $\mathrm{Fpz}$ and $\mathrm{Fz}$ and the ground electrode was placed on the forehead, according to the International 10-20 system. EEG data were filtered online with a bandwidth of $0.1-30 \mathrm{~Hz}$ and electrode impedance was kept constant at $\leq 5 \mathrm{k} \Omega$. EEG estimates were calculated for four frequency bands: delta $(1.5-3.5 \mathrm{~Hz})$, theta $(3.5-7.5 \mathrm{~Hz})$, alpha $(7.5-12-5 \mathrm{~Hz})$ and beta $(12.5-25 \mathrm{~Hz})$, for relative power, and the total power of EEG $(1.5-25 \mathrm{~Hz})$. The most common form of EEG analysis in this study is the calculation of the absolute and relative power estimates.

ERPs were registered and were computed off-line during the performance of the visual and auditory GO/NOGO task. The epoch of analysis included $100 \mathrm{~ms}$ before the first stimulus and $900 \mathrm{~ms}$ after the second stimulus. Trials containing electro-oculogram artefacts (exceeding $100 \mu \mathrm{V}$ thresholds) were discarded in the analysis.

The time in the study was the same for both groups. In the MPH group, medication lasted the same time (4 months). In the NFB group, 40 sessions per child were run 4-month period.

MPH treatment Condition: Prior to achieving a consistent dose of $36 \mathrm{mg} /$ day, participants began the trial with 18 $\mathrm{mg}$ /day for fifteen days, and then increased to $27 \mathrm{mg} /$ day for the following two weeks. Children received one daily dose of $36 \mathrm{mg}$ stimulant prolonged release tablets (Concerta ${ }^{\circledR}$ ) for 3 months administered after breakfast.

NFB treatment Condition: Continuous data from NFB recordings were collected, and training carried out, using the Pro-Comb 2 by Thought Technology hardware and software. The protocol used a mono-polar montage with active electrode cup on the scalp $(\mathrm{Cz})$, the reference cup in the right earlobe (A2) and the ground electrode in left earlobe (A1), according to the International 10-20 system.

The individual sessions was started when participants felt relaxed and were able to maintain normal diaphragmatic breathing, referring to breathing that is done by contracting the diaphragm and give more power to empty lungs. Participants were instructed about the rationale of the procedure and about the dependence of the biofeedback signal on brain activity and on attention.

Protocol and Frequency NFB training

The NFB protocol was dependent on the abnormalities (high theta band measures) observed in the qEEG. In the first phase, children were trained to enhance the amplitude of "sensorimotor rhythm" (SMR, 12-15 Hz) and decrease the amplitude of slow-band theta activity (4-7 Hz). After session number 20 , Beta/theta training was conducted during the second phase and children were instructed to decrease the amplitude of theta waves and increase the amplitude in Beta1 waves $(15-18 \mathrm{~Hz})$. This intervention is based on the wellknown operant conditioning NFB training regimes SMR and "theta-beta" (TB). SMR training is known to reduce hyperactivity and impulsivity, while the beta-1 protocol was maintained to alleviate inattentiveness symptoms. The reward threshold levels were automatically adjusted and the child improved by about $70-80 \%$ with the following duration: 50 minutes and a digitally filtered real-time EEG signal every 30 seconds. The 40 to 45 sessions were held over a period of 15 weeks, three sessions of 60 minutes each per week.

\subsection{Statistical Analysis}

Repeated measures t-tests and ANOVAs were conducted to analyze how behavioral scales and neuropsychological test performance changed after both interventions. The TOVA test measures by ANOVA were corrected by the Bonferroni test.

\section{Results}

\subsection{Effects of NFB Training Compared to the MPH Treatment Group}

Pre and post Behavioral ratings: CPRs/CTRs and BRIEF Parent \& Teachers Scales

A one sample t test showed statistically significant changes in CPRS/CTR and Brief scales as rated by parents and teachers in both conditions (see Table 1). Based on the findings, specific scales were then analyzed (CPRS Parents \& CTRs Teachers: Inattention (In), Hyperactivity/Impulsivity $(\mathrm{H} / \mathrm{I})$, Learning Problems (LP), Executive Functioning (EF), Aggression (A), Peer Relations (PR); BRIEF Parents: Inhibit (I), Shift (S), Emotional Control (EC), Working Memory (WM), Plan/Organize (P/O), Organization of Materials (OM), Monitor (M), Global Executive Composite (GEC); BRIEF Teachers: the form evaluates the same functions except Monitor (M).

A one-sample t-tests showed statistically significant differences in many behavior items in both treatment groups (see Table 2): e.g., CPRs: In (NFB, p =.001; MPH, $\mathrm{p}=.006$ ). In BRIEF Parents: I (NFB, $\mathrm{p}=.024 ; \mathrm{MPH}, \mathrm{p}=.002)$; $\mathrm{S}$ $(\mathrm{NFB}, \mathrm{p}=.001 ; \mathrm{MPH}, \mathrm{p}=.001), \mathrm{EC}(\mathrm{NFB}, \mathrm{p}=.001 ; \mathrm{MPH}$, $\mathrm{p}=.001), \mathrm{WM}(\mathrm{NFB}, \mathrm{p}=.001 ; \mathrm{MPH}, \mathrm{p}=.001), \mathrm{P} / \mathrm{O}(\mathrm{NFB}$, $\mathrm{p}=.003 ; \mathrm{MHP}, \mathrm{p}=.003)$, OM (NFB, $\mathrm{p}=.007 ;$ MHP, $\mathrm{p}$ $=.001), \mathrm{M}(\mathrm{NFB}, \mathrm{p}=.001 ; \mathrm{MPH}, \mathrm{p}=.001)$ and GEC $(\mathrm{NFB}$, $\mathrm{p}=.011 ; \mathrm{MPH}, \mathrm{p}=.003$ ). And in the CTRs (In, NFB, $\mathrm{p}$ $=.001 ; \mathrm{A}, \mathrm{NFB}, \mathrm{p}=.016 ; \mathrm{PR}, \mathrm{NFB}, \mathrm{p}=.017)$. In BRIEF Teachers: $\mathrm{S}(\mathrm{NFB}, \mathrm{p}=.001 ; \mathrm{MPH}, \mathrm{p}=.001)$, EC (NFB, $\mathrm{p}$ $=.001 ; \mathrm{MPH}, \mathrm{p}=.001), \mathrm{WM}(\mathrm{NFB}, \mathrm{p}=.001, \mathrm{MPH}, \mathrm{p}$ $=.001), \mathrm{P} / \mathrm{O}(\mathrm{NFB}, \mathrm{p}=.001 ; \mathrm{MPH}, \mathrm{p}=.003), \mathrm{OM}(\mathrm{NFB}, \mathrm{p}$ $=.001 ; \mathrm{MPH}, \mathrm{p}=.001)$ and $\mathrm{M}(\mathrm{NFB}, \mathrm{p}=.001 ; \mathrm{MPH}, \mathrm{p}$ 
$=.001)$. Notably, the NFB group training group showed statistically significant improvements in some subscales, while the MPH group did not (e.g.: Learning Problems, Executive Functioning and Peer Relations). In contrast, the
MPH group showed statistically significant improvements in some subscales, while the NFB group did not (e.g.: Inhibit in Teacher BRIEF).

Table 1. Description of the groups.

\begin{tabular}{|c|c|c|}
\hline Description of the groups & Neurofeedback $(n=9)$ & Medication $(\mathbf{N}=\mathbf{8})$ \\
\hline Male/Female & $8 / 1$ & $7 / 1$ \\
\hline Age mean (SD) & $10.1(1.3)$ & $9.7(1.0)$ \\
\hline Range (years) & $8.9 / 12.11$ & $8.1 / 12$ \\
\hline Inattentive (fr. \%)* & 22.22 & 25.00 \\
\hline Hyperactive-impulsivity (fr. \%) & 0 & 0 \\
\hline Combined (fr. \%) & 77.77 & 75.50 \\
\hline Motor area: tics, anychophagia (fr. \%) & 11.11 & 12.50 \\
\hline Behavior: obsessive, aggressive, irritable (fr. \%) & 33.33 & 12.50 \\
\hline Social Relations Problem: with parents, sibling, friends (fr. \%) & & 25.00 \\
\hline Emotional Control: anxiety, rigid, ritualistic (fr. \%) & 11.11 & 75.50 \\
\hline Psychosomatics: pain (fr. \%) & 11.11 & 12.50 \\
\hline Language: dyslalias, verbal comprehension deficit, reading and/or written problems (fr. \%) & 66.66 & 75.00 \\
\hline Medication (MPH, Concerta $\left.{ }^{\circledR}\right)$ & no & $36 \mathrm{mg} /$ day \\
\hline
\end{tabular}

Note: *frequency percentage

Table 2. Comparison in Conners Comprehensive Behavior Rating Scales (CPRS/ CTRS) and in the Behavior Rating Inventory of Executive Function (BRIEF) from Parents and Teachers Pre- and Post-treatment standard scores (means + 1SD).

\begin{tabular}{|c|c|c|c|c|c|c|c|c|}
\hline & \multicolumn{4}{|c|}{ Neurofeedback group $(n=9)$} & \multicolumn{4}{|c|}{ MPH group $(n=8)$} \\
\hline & Time $1^{\#}$ & Time $2^{\#}$ & t. & p value & Time $1^{\#}$ & Time $2^{\#}$ & t. & p value \\
\hline \multicolumn{9}{|l|}{ Parents Ratings } \\
\hline \multicolumn{9}{|l|}{ CPRS } \\
\hline Inattention & $79.56(9.35)$ & $56.56(7.16)$ & 5.879 & $0.001 * *$ & $72.50(9.59)$ & $61.00(12.70)$ & 3.880 & $0.006^{*}$ \\
\hline Hyperactivity/Impulsivity & $64.56(19.34)$ & $52.56(8.36)$ & 2.813 & $0.023^{*}$ & $68.00(16.62)$ & $60.50(14.78)$ & 1.651 & 0.143 \\
\hline Learning Problems & $66.44(11.89)$ & $55.78(6.66)$ & 2.619 & $0.031 *$ & $70.75(10.99)$ & $63.00(12.60)$ & 1.802 & 0.115 \\
\hline Executive Functioning & $69.00(13.05)$ & $55.56(7.19)$ & 4.182 & $0.003 *$ & $64.50(11.31)$ & $62.13(11.49)$ & 0.653 & 0.532 \\
\hline Poor Relations & $63.22(9.71)$ & $54.00(6.30)$ & 2.874 & $0.021 *$ & $71.63(14.55)$ & $64.38(13.19)$ & 0.960 & 0.369 \\
\hline \multicolumn{9}{|l|}{ BRIEF } \\
\hline Inhibit & $-1.10(1.33)$ & $0.55(0.52)$ & & 0.353 & $0.00(0.94)$ & $0.62(0.51)$ & & $0.016^{*}$ \\
\hline Shift & $83.33(21.68)$ & $0.55(0.52)$ & & $0.009^{*}$ & $76.62(14.22)$ & $0.62(0.51)$ & & $0.006^{*}$ \\
\hline Emotional Control & $10.00(144.00)$ & $0.44(0.52)$ & & $0.001 * *$ & $10.00(75.28)$ & $0.50(0.53)$ & & $0.002 *$ \\
\hline Initiate & $0.00(1.35)$ & $0.44(0.52)$ & & 0.731 & $0.22(0.82)$ & $0.50(0.53)$ & & 0.427 \\
\hline Working memory & $93.33(20.38)$ & $0.33(0.50)$ & & $0.006^{*}$ & $102.87(12.31)$ & $0.50(0.53)$ & & $0.001 * *$ \\
\hline Org. of Materials & $0.00(1.13)$ & $0.44(0.52)$ & & 0.157 & $0.00(0.48)$ & $1.00(0.00)$ & & 0.072 \\
\hline Monitor & $76.66(17.08)$ & $0.44(0.52)$ & & $0.006^{*}$ & $73.75(7.62)$ & $1.00(0.00)$ & & $0.001 * *$ \\
\hline Global Executive Composite & $6.78(6.01)$ & $0.55(0.52)$ & & 0.116 & $10.51(6.46)$ & $0.55(0.35)$ & & 0.075 \\
\hline \multicolumn{9}{|l|}{ Teachers Ratings } \\
\hline \multicolumn{9}{|l|}{ CTRS } \\
\hline Inattention & $77.67(8.07)$ & $61.00(5.00)$ & & $0.001 * *$ & $69.13(9.76)$ & $63.13(10.81)$ & & 0.259 \\
\hline Hyperactivity/Impulsivity & $60.00(15.74)$ & $55.11(10.71)$ & & 0.449 & $52.25(11.59)$ & $46.50(7.05)$ & & 0.246 \\
\hline Learning/Executive Functioning & $63.89(9.77)$ & $57.11(2.61)$ & & 0.189 & $60.88(10.62)$ & $58.50(5.52)$ & & 0.580 \\
\hline Aggression & $65.33(19.98)$ & $56.78(14.03)$ & & 0.351 & $54.13(13.86)$ & $51.00(8.58)$ & & 0.596 \\
\hline Poor Relations & $66.00(10.42)$ & $61.33(7.10)$ & & 0.537 & $74.63(16.64)$ & $70.75(15.67)$ & & 0.636 \\
\hline \multicolumn{9}{|l|}{ BRIEF } \\
\hline Inhibit & $0.00(0.73)$ & $0.00(0.00)$ & & 0.332 & $0.00(1.13)$ & $0.35(0.51)$ & & 0.117 \\
\hline Shift & $93.66(9.86)$ & $0.00(0.00)$ & & $0.001 * *$ & $83.37(16.98)$ & $0.37(0.51)$ & & $0.007 *$ \\
\hline Emotional Control & $566.88(107.45)$ & $0.00(0.00)$ & & $0.004 *$ & $622.25(91.74)$ & $0.12(0.35)$ & & $0.003 *$ \\
\hline Initiate & $0.33(0.92)$ & $0.00(0.00)$ & & 0.330 & $0.00(0.83)$ & $0.12(0.35)$ & & 0.674 \\
\hline Working memory & $104.66(13.72)$ & $0.00(0.00)$ & & 0.342 & $98.50(12.81)$ & $0.12(0.35)$ & & $0.002 *$ \\
\hline Monitor & $87.00(7.21)$ & $0.00(0.00)$ & & $0.001 * *$ & $80.00(16.10)$ & $0.37(0.51)$ & & $0.007 *$ \\
\hline Global Executive Composite & $3.49(2.80)$ & $0.22(0.44)$ & & 0.099 & $7.59(5.26)$ & $0.62(0.51)$ & & 0.094 \\
\hline
\end{tabular}

Anova Test. $* \mathrm{p}<.0 .5 . * * \mathrm{p}<.01$. 


\subsection{Pre and Post CPT Measurement in Both Groups}

Repeated measures ANOVA showed differences between pre- and post-treatment in both groups in the performance of TOVA (see Table 3). Inattention from the Visual test in NFB group $(p=.001)$ and in Impulsivity and Inattention in MPH group ( $\mathrm{p}=.006, \mathrm{p}=.001$, respectively). Both groups showed statistically significant from pre to post test, in Inattention (In), Impulsivity $(\mathrm{Im})$ and Response Time $(\mathrm{RT})$ from the Auditory test $(\mathrm{In} p=.001 / \mathrm{p}=.001$ respectively; $\mathrm{Im} p=.001 / \mathrm{p}=.001$ respectively; $\mathrm{RT} p=.003 / \mathrm{p}=.008$ respectively).

Table 3. Comparison in Visual and Auditory Test of Variables of Attention (T.O.V.A. $\left.{ }^{\circledR}\right)$ Pre-and Post-treatment standard scores (means $\left.+1 S D\right)$.

\begin{tabular}{|c|c|c|c|c|c|c|c|c|c|c|c|}
\hline \multirow[t]{2}{*}{ Visual TOVA } & \multicolumn{2}{|c|}{ Pre- treatment } & \multicolumn{2}{|c|}{ Post-treatment } & \multirow[b]{2}{*}{$\mathbf{P}$} & \multirow[t]{2}{*}{ Auditory TOVA } & \multicolumn{2}{|c|}{ Pre- treatment } & \multicolumn{2}{|c|}{ Post-treatment } & \multirow[b]{2}{*}{$\mathbf{P}$} \\
\hline & M & SD & M & SD & & & M & SD & $\mathbf{M}$ & SD & \\
\hline Impulsivity & & & & & & Impulsivity & & & & & \\
\hline NFB & 76.33 & 45.59 & 0.33 & 0.50 & 0.055 & NFB & 88.37 & 10.29 & 0.55 & 0.52 & 0.001 \\
\hline $\mathrm{MPH}$ & 87.14 & 17.17 & 0.62 & 0.51 & 0.006 & MPH & 72.12 & 49.73 & 0.37 & 0.51 & 0.001 \\
\hline Inattention & & & & & & Inattention & & & & & \\
\hline NFB & 78.77 & 11.45 & 0.55 & 0.52 & 0.001 & NFB & 214.66 & 51.81 & 0.00 & 0.00 & 0.001 \\
\hline MPH & 82.87 & 18.45 & 0.37 & 0.51 & 0.001 & MPH & 268.00 & 89.78 & 0.37 & 0.51 & 0.001 \\
\hline NFB & -1.11 & 1.63 & 0.77 & 0.44 & 0.442 & NFB & 4.90 & 3.12 & 0.00 & 0.00 & - \\
\hline $\mathrm{MPH}$ & -1.41 & 1.87 & 0.62 & 0.51 & 0.282 & MPH & 6.44 & 4.45 & 0.12 & 0.35 & 0.0634 \\
\hline Response time & & & & & & Response time & & & & & \\
\hline NFB & -0.66 & 1.33 & 0.33 & 0.50 & 0.409 & NFB & 101.0 & 20.24 & 0.44 & 0.52 & 0.003 \\
\hline $\mathrm{MPH}$ & -0.94 & 1.72 & 0.37 & 0.51 & 0.505 & MPH & 88.50 & 24.40 & 0.37 & 0.51 & 0.008 \\
\hline
\end{tabular}

Note: Neurofeedback (NFB), Methylphenidate (MPH), M Mean, SD Standard deviation. Anova Test. * p < . 0.5.** p $<.01$.

\subsection{Pre and Post ERPs Measurement in Both Groups}

In pre - NFB training group, out of 18 ERPs (nine visual and nine auditory, selective attention tasks), 15 (nine visual and six auditory) had abnormal results (delayed latency and reduced amplitude). In the pre - MPH treatment group, out of 16 ERPs (eight visual and eight auditory), 13 (six visual and seven auditory) showed similar abnormal results. In the postNFB training time, four Visual ERPs were normalized (normal parameters for latency and amplitude). In the post NFB group, one auditory ERP was normalized and three obtained normal results in the MPH group (Auditory ERPs Normal $\mathrm{MPH}=42.85 \%$ vs. $\mathrm{NFB}=16.66 \%$ ). In the contrast, in the post - intervention MPH group, four visual ERPs showed more delayed latency compared to the pre-test measures; not negative changes occurred in the NFB group (decline in $\mathrm{ERP}, \mathrm{MPH}=66.6 \%$ vs. $\mathrm{NFB}=0.00 \%$ ).

\subsection{Pre and Post qEEG Measurement in Both Groups}

In the NFB group, eight pre - qEEGs showed abnormal results, while five abnormal results found in the MPH group. In the post - intervention qEEGs of the NFB group, six showed normal results (normal parameters for each age and gender), while only one had normal results in the MPH group $(\mathrm{NFB}=75.00 \%$ vs. $\mathrm{MPH}=20.00 \%)$. In the both groups, abnormal qEEGs showed increased in slow band power activity (an increase in theta, an increased in delta around central and anterior regions and increased in the theta/beta band). No changes were seen in remaining qEEG in either group.

\section{Discussion}

Both conditions, NFB and MPH treatment, proved successful in improving the ADHD symptoms. Both groups showed similar improvements in behavioral and executive functioning rating scales. The effect of NFB training on executive functioning (as rated by parents), and in attention (as rated de by teachers) was greater compared to the MPH group, which showed significant improvement only on the Inhibit subscale of the BRIEF (as rated by parents).

Additionally, we discovered potential variations and clinical differences in TOVAs post - treatments in both groups. In pre - treatment NFB group, eight of the Visual TOVA had abnormal results and only one was normal. At post-test, however, five showed normal results, one had a significant improvement and two did not change. Six of the Auditory TOVA pre - NFBs showed abnormal results. At post-test, four had normal results, one displayed an important and significant improvement and only one did not improve. In the pre- MPH treatment group, seven Visual TOVA and eight of the Auditory TOVA had abnormal results. In the post-MPH treatment, three Visual TOVA showed normal results and four remained abnormal, while three Auditory TOVA had normal results and the rest remained abnormal. We found significant statistically changes in both Auditory TOVA in both groups compared with Visual TOVA changes.

In order to assess whether NFB contributed to sustained improvement on a computerized test of attention and impulse control, participants in this study were retested with the TOVA, four months after their initial evaluation. The TOVA was administered again after both treatment (NFB and MPH) washout periods. As described previously, errors of inattention (i.e., failure to respond to a target stimulus) and impulsivity (i.e., response to a nontarget stimuli), as well as, response rate and the consistency of response rate (variability) were obtained in order to assess the sustained effects of NFB. Standard scores below 80 on any of the 
TOVA subscales are considered to be significantly less than anticipated in individuals with average intelligence (such as our sample). In this study, NFB training of SMR and beta1 band components led to significant general attentionenhancing effect and an arousal-enhancing effect and allowed improve both TOVA modalities and faster reaction times in ERPs latency. For these reasons, in post-SMR and Beta training, measures showed improvements in the visual perceptual sensitivity, fewer Omission and Commission errors, and good performance in inattention and impulsivity. Findings are comparable to those of one study, who used the same NFB training protocol for designed to reduce Theta band activity and increase Beta band activity. And as in our research, one study assessed Attention and impulse control were assessed using one Continuous Performance Test called Integrated Visual Auditory CPT (IVA). His results indicated that the predominantly inattentive group showed significant differences on the Control Scale and the Attention Scale of the IVA/CPT; meanwhile the predominantly hyperactive group showed significant improvement on the Control Scale [48] (Duarte E, 2017). This could directly relate to a improve result in the Visual ERPs. The finding and effects from NFB training in the ERPs performance suggests that NFB promising improve attention in ADHD [17, 19, 49, 50] (Fuchs, Birbaumer, Lutzenberger, Gruzelier, \& Kaiser, 2003; Lubar, 1995; Egner, \& Gruzelier, 2001; 2004).

These results also reflect that electrophysiological training proved improved some concomitant symptoms, such as increased self-attention and positive behavior in children diagnosed with ADHD. Since 2009 several new studies, including 4 placebo-controlled studies, have been published. These latest studies had been review and discussed in more detail. The assessment of specificity of NFB treatment in ADHD was discussed and it concluded that standard protocols such as theta/beta, SMR and slow cortical potentials NFB are well investigated and have demonstrated specificity [51] (Arns, Heinrich, \& Strehl, 2014). After both NFB and MPH treatment, qEEG measures revealed a decrease in delta and theta bands in the anterior and central regions. Two participants showed improved absolute and relative alpha power activity. The percentage of this improvement in cortical arousal was high in NFB group, referring and defining by the non-active presence of slow waves as. During these 4 months of treatment, children and their parents did not receive advice, guidance about developing and practicing active and learning strategies to be implemented in daily life situations. which would optimize the performance effects of this training. The families did not report adverse effects.

Today when we talk about ADHD, we refer to a mild or moderate brain dysfunction that may cause a child or a teen with a normal or greater IQ to display poor academic performance and undesirable behaviors. In other words, despite receiving a good education, having normal family support, support of the person who oversees education, they may not be capable of delivering what is expected of $\mathrm{him} /$ her. For these reasons, appropriate treatment is vital to ensure adequate functioning.

Results from this study suggest additional examination of alternative treatment for ADHD is warranted. Future studies may wish employ strategies that not only have potential to improve symptoms in ADHD, but also offer a warm, encouraging and informative environment.

It is very important to personalized the treatment. Patients with ADHD and families must have the opportunity to be heard. Professionals are encouraged to be able to communicate with patients and their families, in both directions because they not only provide information, and but they should to be responsive to the family's emotions and concerns, and they be able to lead the family to an individualized choice, offer treatment options and avoid making judgments. Participants and their families in both group showed differences in many behavior characteristics, e.g.: in the NFB group, desire and/or motivation for change, enthusiasm to work, interest in learning new skills, were genuine and constant. In contrast, in the MPH group, participants did not show these characteristics; they did not receive support in understanding, confidence and honesty from the neuropsychologist.

Based on the knowledge of Roger deBeus and David Kaiser, it is clear that in the future, additional research with larger sample sizes is needed to aid in identifying differences between those who respond positively to NFB training and those who do not. Larger patients samples may better allow the detection of participants who do not respond well to NFB training and thus determine personalized modifications that may result in improved outcomes, for example, patients may benefit participating in additional NFB training sessions [52] (deBeus, \& Kaiser, 2011).

\section{Conclusions}

NFB training showed greater improvements in specific outcome measures in both neurophysiological markers (qEEG and ERP) and in more behavior and executive functioning subscales (CPRS/CTRS and BRIEF) when compared to the pharmacology MPH group.

Future research should address results in larger samples of children with ADHD for greater generalization ability. Conclusions must await upcoming evidence from larger controlled studies and future meta-analyses contrasting NFB and different outcome measures.

\section{Study Limitations}

This study is not without limitations. First, our small sample size limits the generalizability of findings, and thus these results should be considered preliminary. Further, while the number and frequency of sessions in NFB group (i.e., 40 sessions total three times a week lasting 50 minutes each) were deemed appropriate and successful in improving electrical brain activity. Future studies should consider altering treatment duration and samples sizes, and should be mindful of the fact that ADHD is a heterogeneous disorder 
with several patterns of pathology and different processes that could play a role in successful treatment for children with ADHD.

\section{Competing Interests}

The authors declare that they have no competing interests.

\section{Acknowledgements}

The authors would like to thank the children and their parents for their willingness to participate in this study and Dr. Lynda Thompson for reviewing of the manuscript and her interest. The study was supported by the Consellería de Sanitat i Salut Publica of Valencia (DOCV 6507, 26/04/2011) and was conducted in keeping with the Declaration of Helsinki (World Medical Association, 2013, October). Ethics Committee: 10/061 and 11/083.

\section{References}

[1] Polanczyk, G., de Lima M. S., Horta, B. L., Biederman, J., \& Rohde, L. A. (2007). The worldwide prevalence of ADHD: a systematic review and meta-regression analysis. Am J Psychiatry; 164(6): 942-94. https://ajp.psychiatryonline.org/doi/abs/10.1176/ajp.2007.164. 6.942 .

[2] Mehta, M. A., Goodyer, I. M., \& Sahakian, B. J. (2004). Methylphenidate improves working memory and set-shifting in $\mathrm{AD} / \mathrm{HD}$ : Relationships to baseline memory capacity. $J$ Child Psychol Psychiatry, 45: 293-305.

[3] Klassen, A. F., Miller, A. R., \& Fine, S. (2004). Health related quality of life in children and adolescent who have a diagnosis of attention-deficit/hyperactivity disorder. Pediatrics, 114, 541-547. http://dx.doi.org/ 10.1542/peds.2004-0844.

[4] Monastra, V. J., Lynn, S., Linden, M., Lubar, J. F., Gruzelier, J., \& LaVaque, T. J. (2005). Electroencephalographic biofeedback in the treatment of attention-deficit/hyperactivity disorder. Applied Psychophysiology and Biofeedback, 30, 95114. http://dx.doi.org/ 10.1007/s10484-005-4305-x.

[5] Monastra VJ. (2005). Electroencephalographic biofeedback (neurotherapy) as a treatment for attention deficit hyperactivity disorder: rationale and empirical foundation. Child \& Adolescent Psychiatric Clinics of North America, 14, 55-82. http://dx.doi.org/ 10.1016/j.chc.2004.07.004.

[6] Myer, N. M., Boland, J. R., \& Faraone, S. V. (2017). Pharmacogenetics predictors of methylphenidate efficacy in childhood ADHD. Mol Psychiatry.. doi: 10.1038/mp.2017.234.

[7] Mehta; M. A; Goodyer, I. M, \& Sahakian B. J. (2004). Methylphenidate improves working memory and set-shifting in $\mathrm{AD} / \mathrm{HD}$ : relationships to baseline memory capacity. J Child Psychol Psychiatry. 2004 Feb; 45(2): 293-305.

[8] Kidwell, K. M., Van Dyk, T. R., Lundahl, A. \& Nelson, T. D. (2015). Stimulant Medications and Sleep for Youth With ADHD: A Meta-analysis. Pediatrics, Dec; 136(6): 1144-53. doi: 10.1542/peds.2015-1708.
[9] Barkley, R. A., Fischer, M., Fletcher, K., \& Smallish, L. (2004). Young adult follow-up of hyperactive children: antisocial activities and drug use. Journal of Child Psychology and Psychiatry, http://dx.doi.org/45, 195-211. 10.1111/j.1469-7610.2004.00214.x.

[10] Hammond, D. C. (2011). What is neurofeedback: An update. Journal of Neurotherapy: Investigations in Neuromodulation, Neurofeedback and Applied Neuroscience, 15, 305-336. http://dx.doi.org/10.1080/10874208.2011.623090.

[11] Lubar, J. F. (1997). Neocortial Dynamics: Implications for Understanding the Role of Neurofeedback and Related Techniques for the Enhancement of Attention. Applied Psychophysiology and Biofeedback, 22, 111-126. http://dx.doi.org/10.1023 /A: 1026276228832.

[12] Arns, M., de Ridder, S., Strehl, U., Breteler, M., \& Coenen, A. (2009). Efficacy of neurofeedback treatment in ADHD: the effects on inattention, impulsivity and hyperactivity: a metaanalysis. Clinical EEG and Neuroscience, 40, 180-189. http://dx.doi.org/ 10.1177/155005940904000311.

[13] Magee, C. A., Clarke, A. R., Barry, R. J., McCarthy, R., \& Selikowitz, M. (2005). Examining the diagnostic utility of EEG power measures in children with attention deficit/hyperactivity disorder. Clinical Neurophysiology, 116, 1033-1040. http://dx.doi.org/ 10.1016/j.clinph.2004.12.007.

[14] Rossiter, T. (2004). The effectiveness of neurofeedback and stimulant drugs in treating $\mathrm{AD} / \mathrm{HD}$ : Part I. Review of methodological issues. Applied Psychophysiology and Biofeedback, 29, 95-112. http://dx.doi.org/10.1007/s10484004-0383-4.

[15] Bluschke, A., Broschwitz, F., Kohl, S., Roessner, V., \& Beste, C. (2016). The neuronal mechanisms underlying improvement of impulsivity in ADHD by theta/beta neurofeedback. Sci Rep.; 6: 31178. doi: 10.1038/srep31178.

[16] Beauregard, M., \& Lévesque, J. (2006). Functional magnetic resonance imaging investigation of the effects of neurofeedback training on the neural bases of selective attention and response inhibition in children with attentiondeficit/hyperactivity disorder. Applied Psychophysiology and Biofeedback. http://dx.doi.org/10.1007/s10484-0069001-y.

[17] Fuchs, T., Birbaumer, N., Lutzenberger, W., Gruzelier, J. H., \& Kaiser, J. (2003). Neurofeedback treatment for attentiondeficit/hyperactivity disorder in children: a comparison with methylphenidate. Applied Psychophysiology and Biofeedback, 28, 1-12. http://dx.doi.org/10.1023/A: 1022353731579.

[18] Kropotov, J. D., Grin-Yatsenko, V. A., Ponomarev, V. A., Chutko, L. S., Yakovenko, E. A., \& Nikishena, I. S. (2005). ERPs correlates of EEG relative beta training in ADHD children. International Journal of Psychophysiology, 55, 2334. http://dx.doi.org/10.1016/j.ijpsycho.2004.05.011.

[19] Lubar, J. F., Swartwood, M. O., Swartwood, J. N., \& O'Donnell, P. H. (1995). Evaluation of the effectiveness of EEG neurofeedback training for ADHD in a clinical setting as measured by changes in T. O. V. A. scores, behavioral ratings, and WISC-R performance. Biofeedback and Self Regulation, 20, 83-99. http://dx.doi.org/10.1007/BF01712768.

[20] Nash, J. K. (2000). Treatment of attention deficit hyperactivity disorder with neurotherapy. Clinical EEG, 31, 30-37. http://dx.doi.org/10.1023/A: 1022353731579. 
[21] Thompson, M., Thompson, L. (1998). Neurofeedback combined with training in metacognitive strategies: effectiveness in students with ADD. Applied Psychophysiology and Biofeedback, 23, 243-263. http://dx.doi.org/10.1023/A: 1022213731956.

[22] Fahimi, G., Tabatabaei, S. M., Fahimi, E., \& Rajebi, H. (2017). Index of Theta/Alpha Ratio of the Quantitative Electroencephalogram in Alzheimer's Disease: A Case-Control Study. Acta Med Iran; 55(8): 502-506.

[23] Garn, H., Coronel, C., Waser, M., Caravias, G., \& Ransmayr, G. (2017). Differential diagnosis between patients with probable Alzheimer's disease, Parkinson's disease dementia, or dementia with Lewy bodies and frontotemporal dementia, behavioral variant, using quantitative electroencephalographic features. J Neural Transm (Vienna) ; 124(5): 569-581. doi: 10.1007/s00702017-1699-6.

[24] Haghighi, M., Ludyga, S., Rahimi, B., Jahangard, L., Ahmadpanah, M., Torabian, S.,...\& Brand, S. (2017). In patients suffering from major depressive disorders, quantitative EEG showed favorable changes in left and right prefrontal cortex. Psychiatry Res.; 251: 137-141. doi: 10.1016/j.psychres. 2017.02.012.

[25] Khaleghi, A., Sheikhani, A., Mohammadi, M. R., \& Moti Nasrabadi, A. (2015). Evaluation of Cerebral Cortex Function in Clients with Bipolar Mood Disorder I (BMD I) Compared With BMD II Using QEEG Analysis. Iran J Psychiatry; 10(2): 93-9.

[26] Thatcher, R. W. (2000). EEG operant conditioning (biofeedback) and traumatic brain injury. Clin Electroencephalogr; 31(1): 38-44.

[27] Arns, M., Peters, S., Breteler, R., \& Verhoeven, L. (2007). Different brain activation patterns in dyslexic children: evidence from EEG power and coherence patterns for the double-deficit theory of dyslexia. J Integr Neurosci; 6(1): 17590.

[28] Clarke, A. R., Barry, R. J., McCarthy, R., \& Selikowitz, M. (2001). Age and sex effects in the EEG: differences in two subtypes of attention-deficit/hyperactivity. Clinical Neurophysiology. $\quad$ http://dx.doi.org/112, 815-826. 10.1016/S1388-2457(01)00487-4.

[29] Clarke, A. R., Barry, R. J., McCarthy, R., \& Selikowitz, M. (2001b). Electroencephalogram differences in two subtypes of attention-deficit/hyperactivity disorder. Psychophysiology, 38, 212-221. http://dx.doi.org/10.1111/1469-8986.3820212.

[30] Clarke, A. R., Barry, R. J., McCarthy, R., \& Selikowitz, M. (2001c). Excess beta activity in children with attentiondeficit/hyperactivity disorder: an atypical electrophysiological group. Psychiatry Research, 103, 205-218. http://dx.doi.org/10.1016/S0165-1781(01)00277-3.

[31] Clarke, A. R., Barry, R. J., McCarthy, R., \& Selikowitz, M. (2001d). EEG-defined subtypes of children with attentiondeficit/hyperactivity disorder. Clinical Neurophysiology, 112, 2098-2105. http://dx.doi.org/10.1016/S1388-2457(01)00668$\mathrm{X}$.

[32] Clarke, A. R., Barry, R. J., McCarthy, R., Selikowitz, M., \& Brown, C. R. (2002). EEG evidence for a new conceptualization of attention deficit hyperactivity disorder. Clinical Neurophysiology, 113, 1036-1044. http://dx.doi.org/10.1016/S1388-2457(02)00115-3.
[33] Lazzaro, I., Gordon, E., \& Whitmont, S. (2000). Quantified EEG activity in adolescent attention deficit hyperactivity disorder. Clinical EEG, 29, 37-42. http://dx.doi.org/ $10.1177 / 155005949802900111$.

[34] Hobbs, M. J., Clarke, A. R., Barry, R. J., McCarthy, R., \& Selikowitz, M. (2007). EEG abnormalities in adolescent males with AD/HD. Clinical Neurophysiology, 118, 363-371. http://dx.doi.org/10.1016/j.clinph. 2006.10.013.

[35] Linden, M., Habib, T., \& Radojevic, V. (1996). A controlled study of the effects of EEG biofeedback on cognition and behavior of children with attention deficit disorder and learning disabilities. Biofeedback and Self Regulation, 21, 3549. http://dx.doi.org/ 10.1007/BF02214740.

[36] Fuggetta, G., Bennett, M. A., Duke, P. A., \& Young, A. M. (2014). Quantitative electroencephalography as a biomarker for proneness toward developing psychosis. Schizophr Res.; 153(1-3): 68-77. doi: 10.1016/j.schres. 2014.01.021.

[37] Barry, R. J., Johnstone, S. J., \& Clarke, A. R. (2003). A review of electrophysiology in attention-deficit/hyperactivity disorder: II. Event-related potentials. Clinical Neurophysiology, 114, 184-198. http://dx.doi.org/10.1016/S1388-2457(02)00362-0.

[38] Vernon, D., Egner, T., Cooper, N., Compton, T., Neilands, C., Sheri, A., \& Gruzelier, J. (2003). The effect of training distinct neurofeedback protocols on aspects of cognitive performance. International Journal of Psychophysiology, 47, 75-85. http://dx.doi.org/ 10.1016/S0167-8760(02)00091-0.

[39] Mann, C., Lubar, J. F., Zimmerman, A. W., Millar, C. A., \& Muenchen, R. A. (1992). Quantitative analysis of EEG in boys with attention deficit-hyperactivity. disorder: Controlled study with clinical applications. Pediatriatric Neurology, 8, 30-36. $\mathrm{http} / / / \mathrm{dx}$. doi.org/10.1007/s13311-012-0131-z.

[40] Zhang, D. W., Roodenrys, S., Li, H., Barry, R. J., Clarke, AR.., Wu, Z.,..., \& Sun, L, (2017). Atypical interference control in children with $\mathrm{AD} / \mathrm{HD}$ with elevated theta/ beta ratio. Biol Psychol.; 128: 82-88. doi: 10.1016/j.biopsycho. 2017.07.009.

[41] Loo, S. K., \& Scott, M. (2012). Clinical Utility of EEG in Attention-Deficit/Hyperactivity Disorder: A Research Update. Neurotherapeutics; 9(3): 569-587.doi: 10.1007/s13311-0120131-z.

[42] Barry, R. J., Clarke, A. R., Hajos, M., Dupuy, F. E., McCarthy, R., \& Selikowitz, M. (2011). EEG coherence and symptom profiles of children with Attention-Deficit/Hyperactivity Disorder. Clinical Neurophysiology, 122, 1327-1332. http://dx.doi.org/ 10.1016/j.clinph. 2006.10.002.

[43] Barry, R. J., \& Clarke, A. R. (2013). Resting state brain oscillations and symptom profiles in attention deficit/hyperactivity disorder. Suppl Clin Neurophysiol.; 62: 275-87.

[44] Kröger, A., Hof, K., Krick, C., Siniatchkin, M., Jarczok, T., Freitag, C. M, \& Bender, S. (2014). Visual Processing of Biological Motion in Children and Adolescents with AttentionDeficit/Hyperactivity Disorder: An Event Related PotentialStudy. PLoS One.; 9(2): e88585. doi: 10.1371/journal.pone.0088585.

[45] Gioia, G. A., Isquith, P. K., Guy, S. C, \& Kenworthy, L. (2000). Behavior Rating Inventory of Executive Function (BRIEF). Child Neuropsychology, 6, 235-238. http://dx.doi.org/10.1076/chin.6.3.235.3152. 
[46] Conners, C. (2007). The Conners, 3rd Edition ${ }^{\mathrm{TM}}$. Pearson. Us.

[47] Greenberg, L. M., Kindschi, C. L., \& Corman, C. M. (2000). T. O. V. A. test of variables of attention. Clinical Guide. Copyright Universal Attention Disorders.

[48] Duarte. E., González Marques, J., \& Alvarado, J. M. (2017). Effect of the Theta-Beta Neurofeedback Protocol as a Function of Subtype in Children Diagnosed with Attention Deficit Hyperactivity Disorder. The Spanish Journal of Psychology 2017 | journal-article. DOI: 10.1017/sjp.2016.31.

[49] Egner, T., \& Gruzelier, J. H. (2001). Learned self-regulation of EEG frequency components affects attention and event-related brain potentials in humans. Neuroreport, 21, 4155-4159. http://dx.doi.org/10.1097/00001756-200112210-00058.
[50] Egner, T., \& Gruzelier, J. H. (2004). EEG biofeedback of low beta band components: frequency-specific effects on variables of attention and event-related brain potentials. Clinical Neurophysiology, 115 , 131-139. http://dx.doi.org/10.1016/S1388-2457(03)00353-5.

[51] Arns, M., Heinrich, H., \& Strehl, U. (2014). Evaluation of neurofeedback in ADHD: the long and winding road. Biol Psychol.; 95: 108-15. doi: 10.1016/j.biopsycho. 2013.11.013.

[52] deBeus, R. J., \& Kaiser, D. A. (2011). Neurofeedback with Children with Attentin Deficit Hyperactivity Disorder: A randomized Double-Blind Placebo-Controlled Study. Neurofeedback and Neuromodulation Techniques and $\begin{array}{lll}\text { Applications. } & \text { Elsevier, } & \text { 127-153. }\end{array}$ http://dx.doi.org/10.1007/s00702-010-0524-2. 